\title{
UVA Wahoos at SemEval-2019 Task 6: Hate Speech Identification using Ensemble Machine Learning
}

\author{
Murugesan Ramakrishnan \\ Data Science Institue \\ University of Virginia \\ mr6rxevirginia.edu
}

\author{
Wlodek Zadrozny \\ Computer Science \\ UNC-Charlotte \\ wzadrozn@uncc.edu
}

\author{
Narges Tabari \\ Data Science Institute \\ University of Virginia \\ ns5kn@virginia.edu
}

\begin{abstract}
With the growth in the usage of social media, it has become increasingly common for people to hide behind a mask and abuse others. We have attempted to detect such tweets and comments that are malicious in intent, which either targets an individual or a group. Our best classifier for identifying offensive tweets for SubTask_A (Classifying offensive vs. nonoffensive) has an accuracy of $83.14 \%$ and a f1score of 0.7565 on the actual test data. For SubTask_B, to identify if an offensive tweet is targeted (If targeted towards an individual or a group), the classifier performs with an accuracy of $89.17 \%$ and f1-score of 0.5885 . The paper talks about how we generated linguistic and semantic features to build an ensemble machine learning model. By training with more extracts from different sources (Facebook, and more tweets), the paper shows how the accuracy changes with additional training data.
\end{abstract}

\section{Introduction}

Internet is now accessed by over half of the world's population ${ }^{1}$. In fact, almost 1 million new users are added each day. With social media platforms, people find it a lot easier to get away with the abuse they spew around, in comparison to the offline world. This brings a lot of onus on the Social Network websites to tackle such activities. Majority of the countries have laws to control hate speech which puts tremendous pressure on the concerned websites to curb such activities. Since manual monitoring or defining a specific rule-set might be time consuming, an ensemble machine learning approach has been discussed to avoid complexity and increase interpretability.

The paper focuses on providing solutions to SubTask_A and SubTask_B for the SemEval 2019

\footnotetext{
${ }^{1}$ https://news.itu.int/ itu-statistics-leaving-no-one-offline/
}

competition. Previous works and papers focus on identifying if a tweet is offensive or not. Here, in addition to that, it is identified if an offensive tweet is targeted towards a particular individual or a group (SubTask_B). Such granular information would help the Social Media to make better decisions.

\section{Related Work}

This issue has gathered a lot of attention over the past few years with various types of hate speech detection models.

Papers published in the last two years include the surveys by Schmidt and Wiegand (2017) and Fortuna and Nunes (2018) where the authors extract features from the text like sentiment, linguistic features, utilize different lexical resources to tag an offensive tweet, and another paper by Davidson et al. (2017) presenting the Hate Speech Detection data set used in Malmasi and Zampieri (2017) where the authors perform a three way classification - Hate Speech, Offensive and None. By classifying these, the authors talk about specific patterns related to offensive terms. It is found that the usage of cuss words like $b^{*}$ tch and $n *$ gga is fond in both offensive and casual setting, while $\mathrm{f}^{*}$ ggot and $\mathrm{n}^{*}$ gger were predominantly used in hateful contexts. One of the major takeaways was that lexical methods are effective to identify potentially offensive terms, but are inaccurate at identifying hate speech Other work include: ElSherief et al. (2018); Gambäck and Sikdar (2017); Zhang et al. (2018).

A proposal of typology of abusive language sub-tasks is presented in Waseem et al. (2017) where the author talks about how an offensive tweet can be categorized into four segments - Explicit, Implicit, Directed and Generalized abuse. 
These help in creating segment wise features to capture them separately. Finally, methods in identifying profanity vs. hate speech is talked by Malmasi and Zampieri (2018). This work highlighted the challenges of distinguishing between profanity, and threatening language which may not actually contain profane language.

The description of the current task is presented in detail in Zampieri et al. (2019b), which clearly provides the context and underlying problem statement for this paper.

\section{Data}

For this project, the data set provided by the organizers of OffensEval 2019 was used.The data collection methods used to compile the data set used is described in Zampieri et al. (2019a). The data set consisted of 13,241 records of training observations with the following types of response variables : 1) If a tweet is offensive or not 2) If an offensive tweet is targeted towards an individual (IND) or a group (GRP).

To validate if the performance would increase, an additional data source was also used. The main hypothesis behind including the data was that more data would result in a better accuracy. So, the data set that closely aligned with the current objective was considered for the analysis. This data set was used as a part of the competition organized by TRAC ${ }^{2}$. This contained the response variable with the categories - 'Covertly Aggressive', 'Overtly Aggressive' and 'Non Aggressive'. To maintain consistency with the current data set, 'Covertly Aggressive' and 'Overtly Aggressive' were tagged as 'Offensive' and the rest as 'Not Offensive'.

Including both the data sets, there were a total of 25,239 observations.

The distribution of variables of the original data set is as follows,

SubTask_A: Offensive (33\%), and Nonoffensive (67\%). SubTask_B: Out of the 33\% offensive tweets, it is seen that there are Targeted (88\%), and Untargeted (12\%)

\section{Methodology}

The Methodology involved two sub-works - Feature Engineering and Ensemble Model building.

\footnotetext{
${ }^{2}$ https://sites.google.com/view/trac1/ shared-task/
}

Various features were extracted to get the semantics of the words and tweets.

\subsection{Feature Engineering}

\section{Character n-grams}

Inspired from earlier works, character n-grams were used especially to tackle misspelled words or words without spaces like 'fu*koff' and 'fu*kasdf'. In both of these cases, character 4-gram would detect the sub-word 'fu*k'.

\section{Word n-grams}

Apart from using just 1-gram, 3-gram and 4-gram really helped in identifying the context of the tweet and focus on words like 'not good' where 'not' negates the next word.

\section{Cuss-word Dictionary and Profanity Checker} A list of cuss words were scraped from www.noswearing.com. This helped in identifying such cuss words in tweets which occurred only once or twice in the whole corpus. Profanity checker libraries like profanity were also used along with the scraped list. These helped in creating features like cuss-word count and cuss-word position.

\section{GloVe Embedding}

The use case of GloVe embedding were two-fold. One, average embedding could be found for a tweet which can then be used as a feature space. Two, once the top-30 features were obtained from the initial training, GloVe model was used to find most similar words to them thereby creating a feature representing potential offensive terms.

\section{Part of Speech}

Parts of Speech of the tweets were extracted using spaCy, especially the pronouns which could be used for identifying an individual (SubTask_B).

\section{Others}

Other features like tweet polarity (positive, negative or neural score), of hash-tags, of user tags were also used.

\subsection{Model Building}

Required pre-processing steps like stop-word removal (high and low frequency), stemming, case correction were done. Post which, various features as mentioned above were generated. 


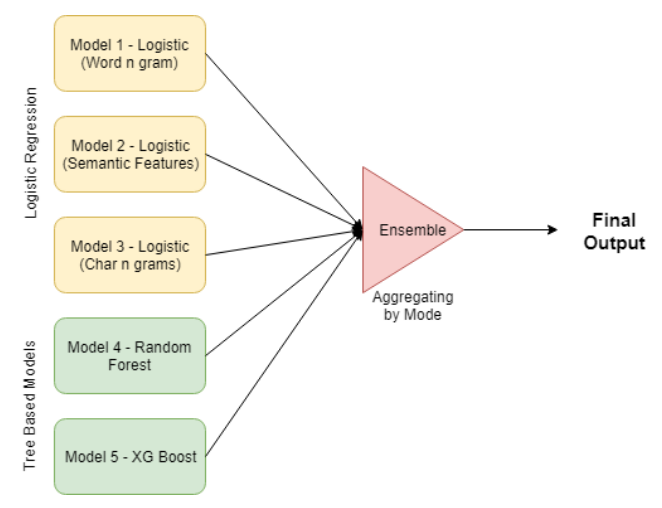

Figure 1: Final Model Architecture

An Ensemble Model was then built by aggregating the results of 5 different models with varying feature set provided as input to each model.

\section{Logistic Regression}

Three basic logistic regression models with L2 regularization were developed:

Model 1 was built with Bag of Words (1 - 4 grams) which amounted to 107,445 number of features

Model 2 was built with Tweet Polarity, Word Embedding, Cuss Word Count, and Cuss Word Position

Model 3 was built with character - 4 grams. Various tests with cross validation were performed to arrive at this result with a final count of 100,122 features

\section{Tree Based Models}

Model 4 was built using Random forest with Bag of Words $(1,2,3,4$ grams) containing a total feature size of 107,237 .

Model 5 using XG Boost, with Bag of Words $(1,2,3,4$ grams) containing a feature size of 107,237 .

The combined architecture looked as follows,

Vote count was made to arrive at the final decision using the outputs from each of these models

\section{Results}

\subsection{Results - SubTask_A}

Model results with respect to the validation data set (part of the training sample) are discussed here. Results of the validation data set with respect to the 80-20 split are shown,

Ensemble Model was able to perform with an accuracy gain of $1.5 \%$ with respect to the best individual model (Model 1)

Similarly, the results for the model using the

\begin{tabular}{|l|c|c|}
\hline \multicolumn{1}{|c|}{ Model } & Accuracy & F1 (macro) \\
\hline Model1 - Logistic (BOW) & 0.78 & 0.73 \\
Model 2- Logistic (Semantic features) & 0.77 & 0.70 \\
Model 3 - Logistic (Char n gram) & 0.76 & 0.71 \\
Model 3 - Random Forest (BOW) & 0.77 & 0.70 \\
Model 4 - XG Boost (BOW) & 0.77 & 0.71 \\
\hline Ensemble Model & $\mathbf{0 . 8 0}$ & $\mathbf{0 . 7 4}$ \\
\hline
\end{tabular}

Table 1: Results for SubTask_A without additional data

given data with an addition of training data provided by TRAC are,

\begin{tabular}{|l|c|c|}
\hline \multicolumn{1}{|c|}{ Model (Additional Data) } & Accuracy & F1 (macro) \\
\hline Model1 - Logistic (BOW) & 0.73 & 0.72 \\
Model 2- Logistic (Semantic features) & 0.70 & 0.69 \\
Model 3 - Logistic (Char n gram) & 0.72 & 0.71 \\
Model 3 - Random Forest (BOW) & 0.71 & 0.69 \\
Model 4 - XG Boost (BOW) & 0.71 & 0.71 \\
\hline Ensemble Model & $\mathbf{0 . 7 4}$ & $\mathbf{0 . 7 3}$ \\
\hline
\end{tabular}

Table 2: Results for SubTask_A with additional data

Comparing the results, it can be seen that addition of data in fact reduces the model accuracy.

\section{Features Analysis- Logistic Regression}

For better intuitive understanding, top features from logistic regression model trained without additional data were extracted to understand what words constitutes a tweet to be offensive,

\begin{tabular}{l|l|}
\hline Variable & Coefficient \\
\hline stupid & 1.798 \\
sucks & 1.513 \\
Cuss_word & 1.453 \\
crap & 1.415 \\
clown & 1.274 \\
idiots & 1.274 \\
bitch & 1.272 \\
sex & 1.231
\end{tabular}

Table 3: Coefficients with higher values

It is clear that words like stupid, sucks, crap and idiots increases the probability of a tweet to be offensive. However, it has been identified that some non-offensive tweets are mis-classified as offensive just because of the presence of such words.

Looking at the coefficients with least weights, it is seen that although the above words have a mild negative connotation, majority of their usecases are not in an offensive setting which makes a tweet with these to have higher probability of non-offensive class.

Validating the results using actual test data: 


\begin{tabular}{|l|l|}
\hline Variable & Coefficient \\
\hline bad & -1.57 \\
mean & -1.08 \\
woman & -1.02 \\
brexit & -0.87 \\
hell & -0.84 \\
fact & -0.76 \\
holy shit & -0.72 \\
pissed & -0.70
\end{tabular}

Table 4: Coefficients with lower values

The results using ensemble model were submitted to the competition and compared against the actual test data. The table shows the baseline results and the model's performance

\begin{tabular}{|lll|}
\hline System & Accuracy & F1 (macro) \\
\hline All NOT baseline & 0.7209 & 0.4189 \\
All OFF baseline & 0.2790 & 0.2182 \\
\hline Ensemble - No additiona data & $\mathbf{0 . 8 3 1 4}$ & $\mathbf{0 . 7 5 6 5}$ \\
Ensemble with additional data & 0.8093 & 0.7433 \\
\hline
\end{tabular}

Table 5: SubTask_A result on actual test data

It was surprising to see that by training the model with additional data, model's accuracy decreased by $3 \%$. This can be mainly attributed to the difference in data sources and difference in response variable definition.

The results between the models trained with and without additional data are to be compared to see the difference between them. Looking at the tweets tagged as Offensive by Model without additional data, but as non-offensive by the other : @USER Zuckerberg lies., SerenaWilliams is so full of herself...she is just as painful to watch as to listen to..., and "50 Cent Calls Out Joe Budden's Bullshit" " On Instagram URL URL. Looking at the tags, it can be hypothesized that words like 'lies','painful' and 'bullshit' which had very high positive score (offensie), got reduced because of the additional data where these words were not used in an offensive setting. Difference in usage of such words is the reason behind reduction in prediction accuracy for new tweets.

\section{Confusion Matrix}

The primary problem is seen with predicting the offensive tweets, where almost half of them are were predicted incorrectly, while a majority of the non-offensive tweets are predicted correctly.

\section{Error Analysis}

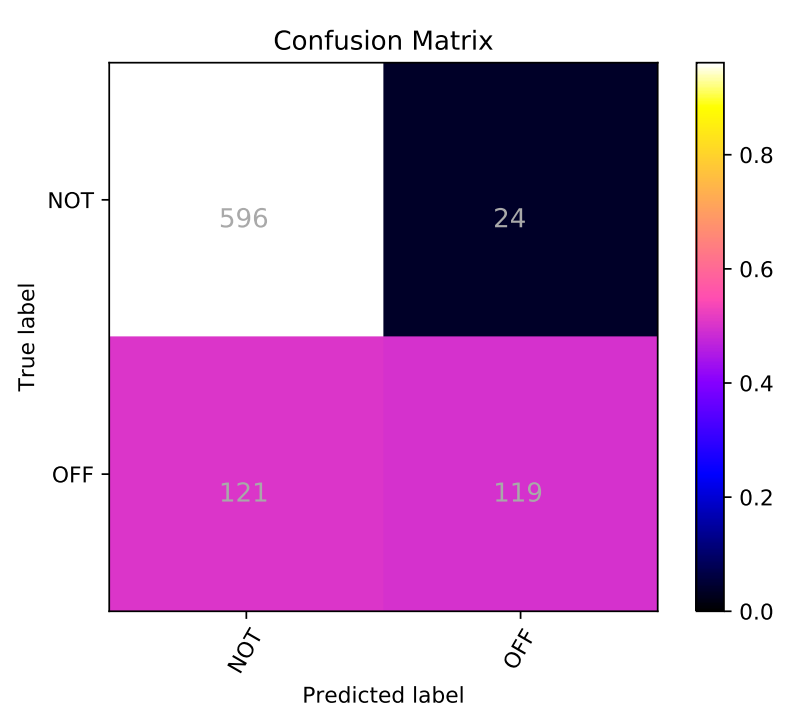

Figure 2: SubTask_A, Ensemble - No additional data

Analyzing the two cases of mis-classification,

i) Offensive tweet tagged non-offensive - While a few of the tweets target an individual or a group, many of them seem to be ambiguous like ”@USER@USER@USER @USER Kick the absolute shite out of the car", "@USER @USER @USER @USER Yes. Yes he is!", and "Shits about to Hit the Fan. MAGA URL"

ii) Non offensive being tagged as offensive The most common reason is the presence of cuss word in a non-offensive sense. Examples are - Am I a dickhead ???? Probably yes,@USER I've already listened to it like 5 times it's so fucking well made More features related to the sequence of the

sentence, and dependency parsing might help in understanding the syntactic structure

\subsection{Results - SubTask_B}

The model architecture remained similar to the earlier SubTask, except that Model 2 was trained with additional features like Parts of Speech to help detect the target.

The cross-validation results obtained are,

\begin{tabular}{|l|c|c|}
\hline \multicolumn{1}{|c|}{ Model (SubTask_B) } & Accuracy & F1 (macro) \\
\hline Model1 - Logistic (BOW) & 0.87 & 0.46 \\
Model 2- Logistic (Semantic features) & 0.87 & 0.46 \\
Model 3 - Logistic (Char n gram) & 0.87 & 0.45 \\
Model 3 - Random Forest (BOW) & 0.86 & 0.46 \\
Model 4 - XG Boost (BOW) & 0.87 & 0.46 \\
\hline Ensemble Model & $\mathbf{0 . 8 8}$ & $\mathbf{0 . 4 7}$ \\
\hline
\end{tabular}

Table 6: Results for SubTask_B with no additional data

It is clear that the ensemble model performs bet- 


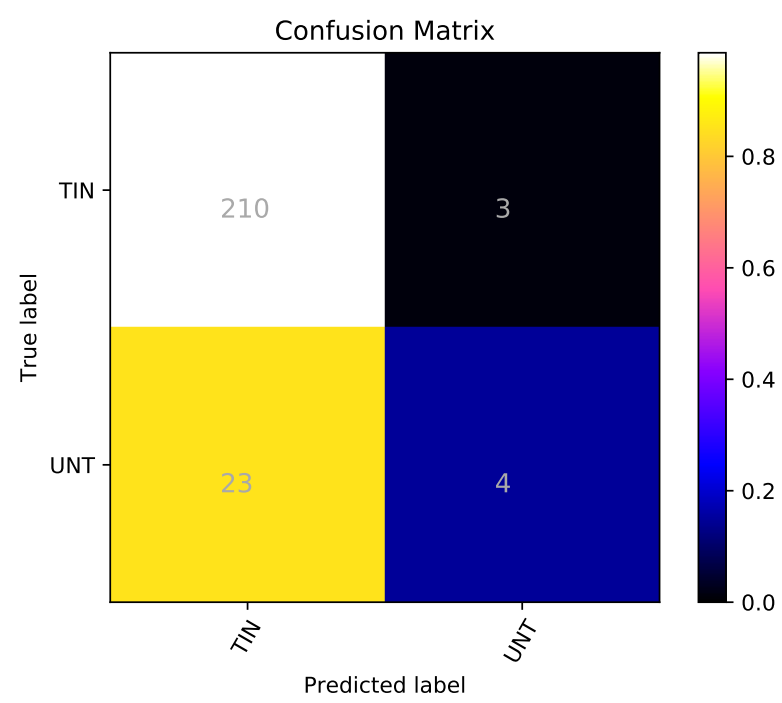

Figure 3: SubTask_B,Confusion matrix for final model

ter than all the other individual models. Now, assessing the performance on actual test data,

\begin{tabular}{|lll|}
\hline System & Accuracy & F1 (macro) \\
\hline All TIN baseline & 0.8875 & 0.4702 \\
All UNT baseline & 0.1125 & 0.1011 \\
\hline Ensemble Model & 0.8917 & 0.5885 \\
\hline
\end{tabular}

Table 7: SubTask_B result on actual test data

\section{Confusion Matrix}

Looking at the confusion matrix in Figure 3, the main issue is seen with predicting a targeted tweet, where almost all of them are predicted incorrectly. This can be ascribed to insufficient number of features that would identify an individual or organization.

\section{Conclusion}

The proposed ensemble model leverages the best of each of the individual models, where each of the models was experimented with a varying set of features. Some features like word and character n-grams, tweet polarity, cuss word count were more helpful in capturing offensive tweets. The performance for SubTask_B is not appreciable because less number of features related to identifying a target were used. With our best scores for SubTask_A, we were placed 36th out of 103 participants, and were placed 42 nd out of 75 submissions in the SemEval-2019 competition. The top team achieved a F1(macro) score of 0.829 in SubTask_A, while we obtained 0.756. Similarly, for SubTask_B the top team had a F1(macro) score of 0.755 , while we obtained 0.588 . Features like identifying the presence of a person's name using nltk libraries, and the presence of an individual or an organization using Named Entity Recognition with spaCy is highly recommended for further studies.

Moreover, there was a pattern associated with the coefficients having least weights - most of them had a slightly negative connotation. This can justified because of the overall theme of tweets used for training, as most of them were inclined towards politics. This lead to overall less number of 'positive words'. Having more training examples especially with a range of tweet polarity from more positive to more negative would help in building better models. As seen from our results, an additional data set should be in the same space (Twitter data) to avoid worse performance.

Talking about the offensive tweet categories, there are four types - explicit, implicit, targeted at individuals and groups. The techniques mentioned in this paper using a variety of feature engineering tries to capture most of these categories. However, advanced syntactic features should be introduced to capture patterns like "Pronoun-VerbCuss_word". Especially, if a exploratory data analysis is performed on analyzing the patterns of Parts of Speech in offensive tweets, it would help in building additional useful variables.

The main goal of this paper is to show that simpler models which have understandable features can produce good results. More complex methods like introducing polynomial or intricate features, deep learning models using Recurrent Neural Network are other approaches for potentially better accuracy but at the risk of losing interpretability. In conclusion, it is believed that with additional robust features as discussed earlier, the current ensemble machine learning model's performance might increase. Moreover, such features would also be really helpful in interpreting why a tweet could be offensive, which will help in taking necessary actions and remedial measures for social media companies.

\section{References}

Thomas Davidson, Dana Warmsley, Michael Macy, and Ingmar Weber. 2017. Automated Hate Speech Detection and the Problem of Offensive Language. In Proceedings of ICWSM.

Mai ElSherief, Vivek Kulkarni, Dana Nguyen, 
William Yang Wang, and Elizabeth Belding. 2018. Hate Lingo: A Target-based Linguistic Analysis of Hate Speech in Social Media. arXiv preprint arXiv:1804.04257.

Paula Fortuna and Sérgio Nunes. 2018. A Survey on Automatic Detection of Hate Speech in Text. ACM Computing Surveys (CSUR), 51(4):85.

Björn Gambäck and Utpal Kumar Sikdar. 2017. Using Convolutional Neural Networks to Classify Hatespeech. In Proceedings of the First Workshop on Abusive Language Online, pages 85-90.

Shervin Malmasi and Marcos Zampieri. 2017. Detecting Hate Speech in Social Media. In Proceedings of the International Conference Recent Advances in Natural Language Processing (RANLP), pages 467472.

Shervin Malmasi and Marcos Zampieri. 2018. Challenges in Discriminating Profanity from Hate Speech. Journal of Experimental \& Theoretical Artificial Intelligence, 30:1-16.

Anna Schmidt and Michael Wiegand. 2017. A Survey on Hate Speech Detection Using Natural Language Processing. In Proceedings of the Fifth International Workshop on Natural Language Processing for Social Media. Association for Computational Linguistics, pages 1-10, Valencia, Spain.

Zeerak Waseem, Thomas Davidson, Dana Warmsley, and Ingmar Weber. 2017. Understanding Abuse: A Typology of Abusive Language Detection Subtasks. In Proceedings of the First Workshop on Abusive Langauge Online.

Marcos Zampieri, Shervin Malmasi, Preslav Nakov, Sara Rosenthal, Noura Farra, and Ritesh Kumar. 2019a. Predicting the Type and Target of Offensive Posts in Social Media. In Proceedings of NAACL.

Marcos Zampieri, Shervin Malmasi, Preslav Nakov, Sara Rosenthal, Noura Farra, and Ritesh Kumar. 2019b. SemEval-2019 Task 6: Identifying and Categorizing Offensive Language in Social Media (OffensEval). In Proceedings of The 13th International Workshop on Semantic Evaluation (SemEval).

Ziqi Zhang, David Robinson, and Jonathan Tepper 2018. Detecting Hate Speech on Twitter Using a Convolution-GRU Based Deep Neural Network. In Lecture Notes in Computer Science. Springer Verlag. 